\title{
Wavelet primal sketch representation using Marr wavelet pyramid and its reconstruction
}

\author{
Dimitri Van De Ville ${ }^{1,2}$, Michael Unser ${ }^{1}$ \\ ${ }^{1}$ École Polytechnique Fédérale de Lausanne (EPFL), Switzerland \\ ${ }^{2}$ University of Geneva, Switzerland
}

\begin{abstract}
Based on the class of complex gradient-Laplace operators, we show the design of a non-separable two-dimensional wavelet basis from a single and analytically defined generator wavelet function. The wavelet decomposition is implemented by an efficient FFT-based filterbank. By allowing for slight redundancy, we obtain the Marr wavelet pyramid decomposition that features improved translation-invariance and steerability. The link with Marr's theory of early vision is due to the replication of the essential processing steps (Gaussian smoothing, Laplacian, orientation detection). Finally, we show how to find a compact multiscale primal sketch of the image, and how to reconstruct an image from it.
\end{abstract}

Keywords: Multidimensional wavelet design, Laplacian-of-Gaussian, complex gradient, Wirtinger calculus, pyramid decomposition, raw primal sketch

\section{INTRODUCTION}

Multiscale transforms are prominent tools for signal and image processing, computer vision, and modeling of biological vision. Most notably, the 1-D wavelet transform acts as a multiscale version of an $N$ th-order derivative operator, where $N$ is the number of vanishing moments of the wavelet. ${ }^{1}$ The extension to multiple dimensions and to 2-D, in particular, is typically obtained by tensor-product basis functions. Considerable research effort has been invested in developing alternative multiscale transforms that are better tuned to the geometry of natural images. Some examples of these "geometrical x-lets" include biologically-inspired 2-D Gabor transforms, ${ }^{2}$ wedgelets, ${ }^{3}$ ridgelets,,${ }^{4,5}$ dual-tree constructions ${ }^{6,7}$ and the $M$-band extension, ${ }^{8}$ curvelets,,${ }^{9,10}$ contourlets, ${ }^{11}$ bandelets, ${ }^{12,13}$ directional wavelet frames, ${ }^{14}$ and directionlets. ${ }^{15}$ Another important class of multiscale transforms are 2-D directional filterbanks ${ }^{16}$ and steerable pyramids, ${ }^{17-19}$ which combine the structure of the Laplacian pyramid ${ }^{20}$ with steerable filters. ${ }^{21,22}$

Here, we follow an alternative design strategy. From the complex gradient-Laplace operator, we define associated complex polyharmonic B-splines. The spline spaces then form embedded approximation spaces, from which we can define a wavelet basis that spans the orthogonal complement. A particular feature of our approach is that the wavelets are defined from a single-generator wavelet. Next, we extend the wavelet basis to a mildlyredundant pyramid structure by not subsampling the wavelet subband and exploiting the fact that a single generator wavelet is used. Moreover, we benefit from the enlarged wavelet space to further shape the wavelet function; i.e., the smoothing kernel within the wavelet is chosen to closely resemble a Gaussian. Due to the close match of the processing elements with the ones proposed by Marr for modeling early vision, ${ }^{23}$ we coin the term "Marr wavelet pyramid" for this decomposition. We also derive a compact multiscale primal sketch. Finally, we propose an efficient image reconstruction algorithm given its primal sketch representation.

Further author information: (dimitri.vandeville, michael.unser)@epfl.ch

Wavelets XIII, edited by Vivek K. Goyal, Manos Papadakis, Dimitri Van De Ville, Proc. of SPIE Vol. 7446, 74460W · @ 2009 SPIE · CCC code: 0277-786X/09/\$18 · doi: 10.1117/12.825972 


\section{OPERATOR-LIKE WAVELET BASES}

\subsection{Complex Polyharmonic B-splines}

The complex gradient-Laplace operators form a class of differential operators that are most appealing for image processing. They can be characterized as

$$
\mathrm{L}_{\gamma, N}=(-\Delta)^{\frac{\gamma-N}{2}}\left(-j \frac{\partial}{\partial x_{1}}-\frac{\partial}{\partial x_{2}}\right)^{N}
$$

where $\gamma \in \mathbb{R}^{+}$and $N \in \mathbb{N}$. This is the complete family of complex operators that combines translation-invariance, scale-invariance, and rotation-covariance. ${ }^{24}$

Associated to this class of operators, complex polyharmonic B-splines are non-separable basis functions that are defined in the Fourier domain as

$$
\hat{\beta}_{\gamma, N}=\frac{V_{\gamma, N}\left(e^{j \omega}\right)}{\hat{L}_{\gamma, N}(\boldsymbol{\omega})},
$$

where the numerator $V_{\gamma, N}\left(e^{j \boldsymbol{\omega}}\right)$ is the localization filter, and the denominator $\hat{L}_{\gamma, N}(\boldsymbol{\omega})=\|\boldsymbol{\omega}\|^{\gamma-N}\left(\omega_{1}-j \omega_{2}\right)^{N}$ is the Fourier transform of (1) in the distributional sense.

The localization filter regularizes the singularity of the Fourier transform at the origin. Let us consider the polar-separable representation $V_{\gamma, N}\left(e^{j \boldsymbol{\omega}}\right)=V_{\gamma}\left(e^{j \boldsymbol{\omega}}\right) \cdot e^{j \theta_{N}(\boldsymbol{\omega})}$ where we specify modulus and phase as

$$
\begin{aligned}
V_{\gamma}\left(e^{j \boldsymbol{\omega}}\right) & =\left(\frac{8}{3}\left(\sin ^{2}\left(\frac{\omega_{1}}{2}\right)+\sin ^{2}\left(\frac{\omega_{2}}{2}\right)\right)+\frac{2}{3}\left(\sin ^{2}\left(\frac{\omega_{1}+\omega_{2}}{2}\right)+\sin ^{2}\left(\frac{\omega_{1}-\omega_{2}}{2}\right)\right)\right)^{\gamma / 2}, \\
\theta_{N}(\boldsymbol{\omega}) & =\angle\left(j\left[\omega_{1}\right]-j\left[\omega_{2}\right]\right)^{N},
\end{aligned}
$$

and where $[\omega]$ stands for the unique $\omega$ in $[-\pi, \pi[$ such that $\omega-[\omega]=2 n \pi$ for some integer $n$. The construction makes $\beta_{\gamma, 0}$ coincide with the isotropic polyharmonic B-splines ${ }^{25}$ that were earlier introduced as extensions of the elementary polyharmonic B-splines. ${ }^{26}$ Thanks to the property $\left|\hat{\beta}_{\gamma, N}\right|=\hat{\beta}_{\gamma, 0}$, the autocorrelation filter

$$
A_{\gamma}\left(e^{j \omega}\right)=\sum_{\mathbf{k}}\left|\hat{\beta}_{\gamma}(\boldsymbol{\omega}+2 \pi \mathbf{k})\right|^{2}
$$

does not depend on $N$. The interpolant $\phi_{2 \gamma}$ of the spline space* associated to $\mathrm{L}_{\gamma, N}^{*} \mathrm{~L}_{\gamma, N}$ is also independent of $N$; its Fourier transform is given by

$$
\hat{\phi}_{2 \gamma}(\boldsymbol{\omega})=\frac{\left|\hat{\beta}_{\gamma}(\boldsymbol{\omega})\right|^{2}}{A_{\gamma}\left(e^{j \boldsymbol{\omega}}\right)} .
$$

\subsection{Complex Wavelet Basis}

The complex polyharmonic B-splines are valid scaling functions to build a multiscale analysis of $L_{2}\left(\mathbb{R}^{2}\right):{ }^{27}$ their integer shifts form a Riesz basis; they satisfy the partition of unity property; and they satisfy a scaling relation for a subsampling matrix $\mathbf{D}$ that corresponds to a rotation combined with a dilation. Specifically, in the Fourier domain, the scaling relation can be described as

$$
\hat{\beta}_{\gamma, N}\left(\mathbf{D}^{T} \boldsymbol{\omega}\right)=|\operatorname{det} \mathbf{D}|^{-\frac{1}{2}} H\left(e^{j \boldsymbol{\omega}}\right) \hat{\beta}_{\gamma, N}(\boldsymbol{\omega}),
$$

where $H\left(e^{j \omega}\right)$ is the scaling filter. We consider the embedded approximation spaces

$$
\mathcal{V}_{i}=\operatorname{span}_{\mathbf{k} \in \mathbb{Z}^{2}}\left\{|\operatorname{det} \mathbf{D}|^{\frac{i}{2}} \beta_{\gamma, N}\left(\mathbf{D}^{i} \mathbf{x}-\mathbf{k}\right)\right\}
$$

to construct wavelets that span the orthogonal complements $\mathcal{W}_{i}=\mathcal{V}_{i} \ominus_{\perp} \mathcal{V}_{i-1}$. The approximation coefficients at scale $i$ are denoted as $c_{i}[\mathbf{k}]$.

\footnotetext{
*This "augmented order" polyharmonic space is spanned by $\beta_{\gamma, N}(\cdot) * \beta_{\gamma, N}(-\cdot)=\beta_{2 \gamma, 0}$.
} 


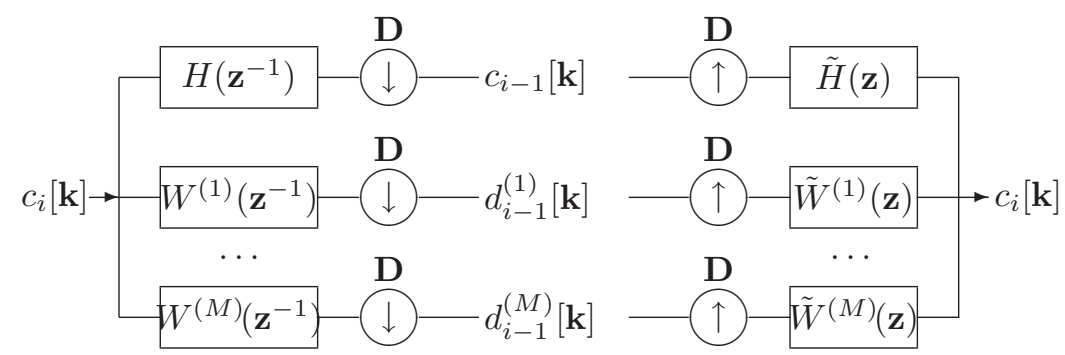

(a)

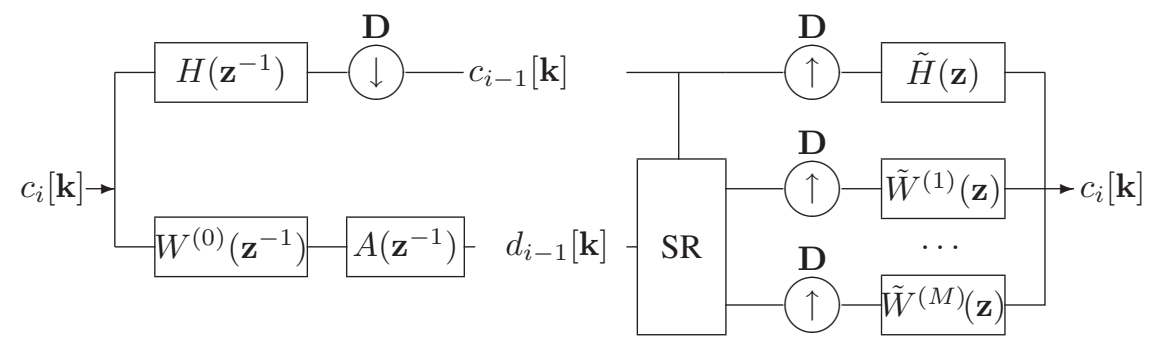

(b)

Figure 1. (a) Filterbank corresponding to the operator-like wavelet decomposition. (b) Filterbank corresponding to the Marr wavelet pyramid. The synthesis is performed by the "subband regression" (SR) algorithm that projects the redundant representation back into the basis.

\subsection{Operator-Like Wavelets}

One particular difficulty in multidimensional wavelet design is the choice of the $M=|\operatorname{det} \mathbf{D}|-1$ wavelets that span the wavelet subspace. We propose "operator-like" wavelets: a single wavelet generator is shifted on $M$ positions of the cosets defined by $\mathbf{D}$. Specifically, we define the wavelets that span the detail space $\mathcal{W}_{-1}$ as

$$
\psi_{\gamma, N}^{(n)}\left(\mathbf{D}^{-1} \mathbf{x}\right)=(-\Delta)^{\frac{\gamma-N}{2}}\left(j \frac{\partial}{\partial x_{1}}-\frac{\partial}{\partial x_{2}}\right)^{N}\left\{\phi_{2 \gamma}\right\}\left(\mathbf{x}+\mathbf{e}_{n}\right), \quad n=1, \ldots, M,
$$

where the smoothing kernel $\phi_{2 \gamma}$ is specified by (4) and $\mathbf{e}_{n}$ is in the cosets $\mathcal{C} \backslash \mathbf{e}_{0}=(0,0)$ of the subsampling matrix D. By writing the generator wavelet as $\psi_{\gamma, N}^{(0)}=\psi_{\gamma, N}$, we can compactly denote the wavelet subspaces $\mathcal{W}_{i}$ as

$$
\mathcal{W}_{i}=\operatorname{span}\left\{|\operatorname{det}(\mathbf{D})|^{i / 2} \psi_{\gamma, N}\left(\mathbf{D}^{i} \mathbf{x}-\mathbf{D}^{-1} \mathbf{k}\right)\right\}_{\mathbf{k} \in \mathbb{Z}^{2} \backslash \mathbf{D} \mathbb{Z}^{2}} .
$$

We have proven ${ }^{24}$ that operator-like wavelets generate a semi-orthogonal basis of $L_{2}\left(\mathbb{R}^{2}\right)$.

Practically, the wavelet decomposition is implemented using a filterbank. The wavelet filters can be obtained by expressing $\psi_{\gamma, N}^{(n)}$ as a function of the scaling function $\beta_{\gamma, N}$ :

$$
\hat{\psi}_{\gamma, N}^{(n)}\left(\mathbf{D}^{T} \boldsymbol{\omega}\right)=|\operatorname{det} \mathbf{D}|^{-1} \underbrace{\frac{V_{\gamma, N}^{*}\left(e^{j \boldsymbol{\omega}}\right)}{A_{\gamma}\left(e^{j \boldsymbol{\omega}}\right)} e^{j \boldsymbol{\omega}^{T} \mathbf{e}_{n}}}_{W^{(n)}\left(e^{j \boldsymbol{\omega}}\right)} \hat{\beta}_{\gamma, N}(\boldsymbol{\omega}) .
$$

In Fig. 1 (a), we show the filterbank that is used to implement the wavelet transform. The dual filters at the synthesis side can be determined by deriving the scaling relations for the (unique) dual functions, or, by expressing the perfect reconstruction condition on the filters. Notice that the wavelet coefficients are complex-valued. From now on, we restrict ourselves to the dyadic subsampling scheme $\mathbf{D}=\operatorname{diag}(2,2)$. 


\section{MARR WAVELET PYRAMID}

\subsection{Marr's Theory of Vision}

The complex gradient-Laplace operator has been widely applied in image processing. Moreover, as proposed by David Marr in his seminal work on vision, ${ }^{23}$ it also plays a central role in the modeling of the early stage of the primate's visual system. In his framework, image smoothing by a Gaussian, application of the Laplacian operator, and detecting zero-crossing with their orientation, are identified as basic operations of low-level vision. As an outcome of these basic operations, Marr introduced the concept of the "raw primal sketch". This theory has highly influenced the fields of vision and image processing; e.g., scale-space theory. ${ }^{28-30}$ Reconstructing back an image that closely matches the original one from its (multiscale) primal sketch representation has been a long standing challenge. It is related to the inversion problem from zero-crossings that has been addressed for signals ${ }^{31-34}$ and for Laplacian-filtered images. ${ }^{35,36}$ Most interestingly for our purpose, Mallat ${ }^{37}$ investigated this problem using the (fully redundant) wavelet transform. In the improved algorithm, ${ }^{38}$ Mallat and Zhong reconstruct using the positions and the values of the modulus maxima of the wavelet coefficients. Two separate fully redundant wavelet transforms are required.

\subsection{Marr Wavelet Pyramid}

The operator-like wavelet decomposition can be tuned to match the basic operatorions in Marr's model in a multiscale fashion.

- First, we select the operator $\mathrm{L}_{\gamma, N}$ with $\gamma=3, N=1$; i.e., the pure Laplacian is combined with the complex gradient.

- Second, since the three wavelets $\psi_{3,1}^{(n)}, n=1, \ldots, 3$ originate from the same generator function $\psi_{3,1}$, we can rearrange them in a single subband and add the "missing" shift (for $\mathbf{e}_{0}=\left[\begin{array}{ll}0 & 0\end{array}\right]^{T}$ ); i.e., we do not subsample the wavelet subband after filtering with $W^{(0)}\left(e^{j \omega}\right)$. The enlarged wavelet spaces can be denoted as

$$
\mathcal{W}_{i}^{+}=\operatorname{span}\left\{\psi_{3,1}\left(2^{i} \mathbf{x}-\mathbf{k} / 2\right)\right\}_{\mathbf{k} \in \mathbb{Z}^{2}}
$$

and the (non-subsampled) wavelet coefficients at scale $i$ as $d_{i}[\mathbf{k}]$. In this slightly redundant scheme, the reconstruction of the pyramid decomposition is performed using the subband regression algorithm; i.e., the pyramid data is reprojected onto the (non-redundant) wavelet basis such as to minimize a quadratic consistency error criterion. ${ }^{39}$ This extension improves the shift-invariance of the decomposition and allows conventional image processing algorithms to be deployed "in-band".

- Third, the smoothing kernel $\phi_{2 \gamma}$, which is an interpolant, can be changed to better resemble the Gaussian using the additional freedom from the enlarged wavelet space. Specifically, we filter the wavelet coefficients with $A\left(e^{-j \omega}\right)$, which turns $\phi_{2 \gamma}$ into $\beta_{2 \gamma}$. The latter function is known to converge to a Gaussian as $\gamma$ increases. ${ }^{25}$ The generator wavelet can now be written as

$$
\psi(\mathbf{x} / 2)=\Delta\left(j \frac{\partial}{\partial x_{1}}-\frac{\partial}{\partial x_{2}}\right)\left\{\beta_{2 \gamma}\right\}(\mathbf{x}) .
$$

Thanks to the Gaussian-like smoothing kernel, the Marr wavelet (11) becomes quasi-steerability; i.e., $\operatorname{Re}\{\psi\}$ can be rotated at any angle by a suitable combination with $\operatorname{Im}\{\psi\}$.

The filterbank implementation of the wavelet pyramid is shown in Fig. 1 (b). The redundancy factor is only $4 / 3$, bringing the total redundancy (including the factor 2 for the complex-valued coefficients) to $8 / 3$. 


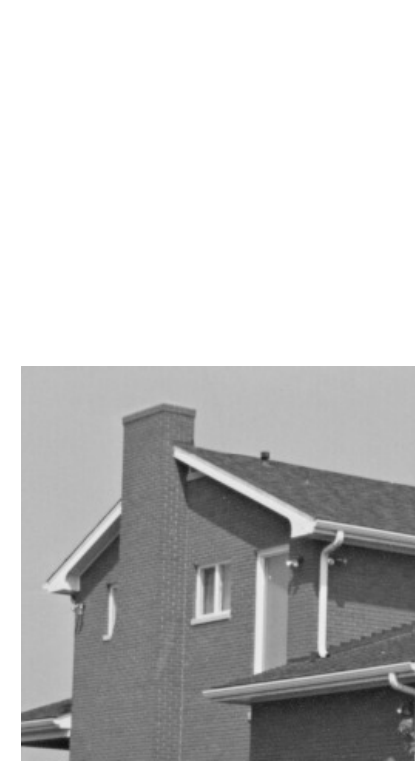

(a)
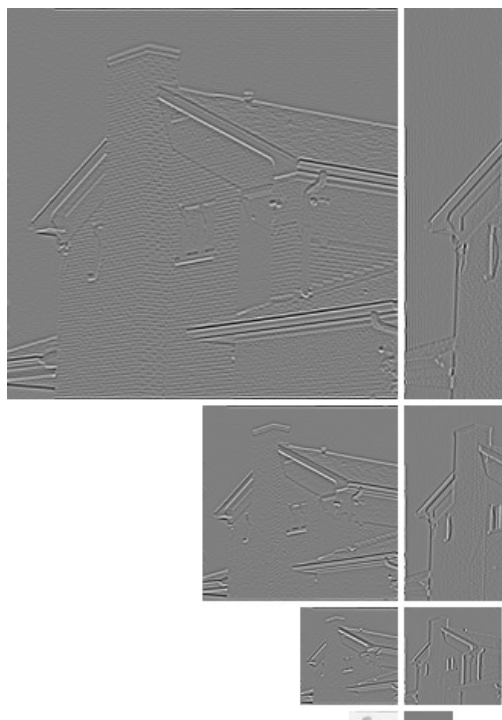

国

(b)

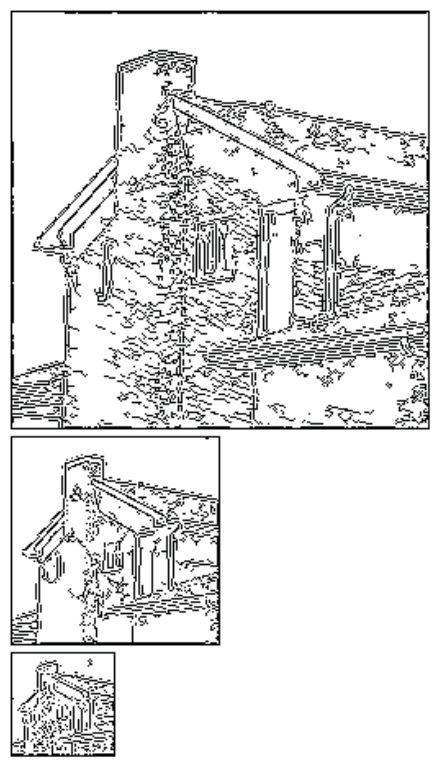

(c)

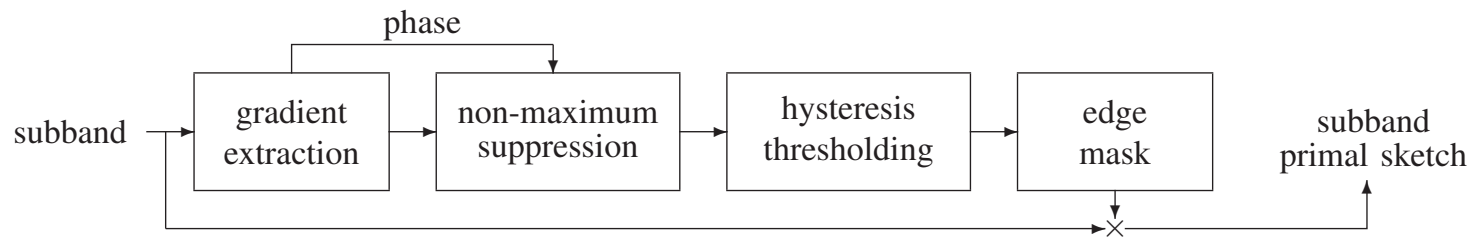

(d)

Figure 2. (a) Test image "house". (b) Marr wavelet pyramid. (c) Wavelet primal sketch. The map indicates which coefficients are part of the sketch representation. (d) Flowchart of how the sketch is obtained for each subband.

\subsection{Wavelet Primal Sketch and Its Reconstruction}

We are able to obtain the wavelet primal sketch by applying an Canny-like edge detection. As schematically shown in Fig. 2 (d), the local gradient information can be extracted from the real and imaginary parts of the wavelet coefficients. Next, non-maximum suppression along the direction perpendicular to the local edge and hysteresis thresholding allow to extract a robust edge map. In (b), the Marr wavelet pyramid for the test image in (a) is shown, and the map indicating the constituting coefficients of the wavelet primal sketch in (c).

Next, we propose a reconstruction procedure that is inspired by the one proposed by Mallat and Zhong. Practically, our algorithm uses alternating projections between two spaces:

- First, we define the space $\mathcal{M}$ of all Marr pyramid transforms of real-valued functions in $L^{2}\left(\mathbb{R}^{2}\right)$. Any such Marr pyramid transform is invariant under the projector

$$
P_{\mathcal{M}}=W \odot \operatorname{Re} \odot W^{-1},
$$

which consists of three operations: the inverse wavelet transform $W^{-1}$ is an orthogonal projector onto the image with respect to the norm that is minimized by the subband regression algorithm; the pointwise transform Re is an orthogonal projector onto the space of real-valued images; and the wavelet transform $W$ provides the Marr wavelet pyramid of the new image.

- Second, we want the Marr pyramid that has the same values on the domain $\Omega_{i}$ of the wavelet primal sketch. We search for a solution that is consistent with the wavelet primal sketch (i.e., the desired values $d_{i}[\mathbf{k}]$ on $\Omega_{i}$ ) and that minimizes some ad-hoc wavelet-domain energy. Specifically, we would like the coefficients to 


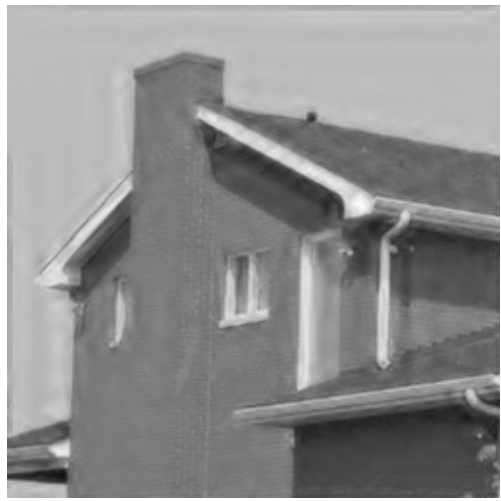

(a)

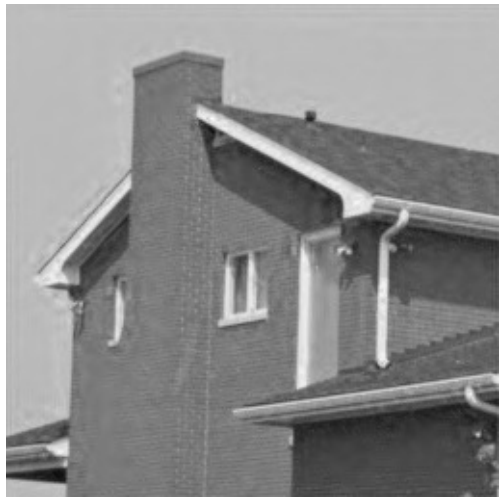

(b)

Figure 3. Reconstruction from the wavelet primal sketch. (a) Result after one iteration. (b) Result after 30 iterations (PSNR 35.8dB).

be small and essentially non-oscillating. Therefore, we propose to minimize the regularizing norm

$$
\left|\left(d_{i}[\mathbf{k}]\right)_{i, \mathbf{k}}\right|_{R}^{2}=\sum_{i \in \mathbb{Z}} \sum_{\mathbf{k} \in \mathbb{Z}^{2}}\left|d_{i}[\mathbf{k}]\right|^{2}+\lambda_{i}\left(\left|d_{i}[\mathbf{k}]-d_{i}\left[\mathbf{k}-\mathbf{e}_{1}\right]\right|^{2}+\left|d_{i}[\mathbf{k}]-d_{i}\left[\mathbf{k}-\mathbf{e}_{2}\right]\right|^{2}\right),
$$

where $\mathbf{e}_{1}$ and $\mathbf{e}_{2}$ are the unit horizontal and vertical vectors, respectively. Let us define $\mathcal{R}$ as the space of pyramids with a consistent primal sketch; i.e., $\left(d_{i}\right)_{i, \mathbf{k}}$ such that $\left(\left.d_{i}\right|_{\Omega_{i}}\right)$ match. A critical component of our algorithm is the projector $P_{\mathcal{R}}$ onto $\mathcal{R}$, which is orthogonal with respect to the norm (13), and which is specified in detail in Appendix A.

The reconstruction algorithm can now be formalized as the search for an image corresponding to an admissible Marr wavelet pyramid such that

$$
\left(d_{i}^{\prime}[\mathbf{k}]\right)_{i, \mathbf{k}} \in \mathcal{M}, \text { subject to } \min _{\left(d_{i}^{\prime} \mid \Omega_{i}\right)=\left(d_{i} \mid \Omega_{i}\right)}\left|\left(d_{i}^{\prime}[\mathbf{k}]\right)_{i, \mathbf{k}}\right|_{R}^{2},
$$

where $\left(\left.d_{i}\right|_{\Omega_{i}}\right)$ is the given wavelet primal sketch. This objective can be reached by alternating between the projectors $P_{\mathcal{M}}$ and $P_{\mathcal{R}}$, a procedure that is proven to converge to the orthogonal projection on $\Lambda=\mathcal{M} \cap \mathcal{R} .{ }^{40}$ If we start from the zero element of $\mathcal{M}$, we are eventually going to converge to the element of $\Lambda$ whose norm (13) is minimum.

In Fig. 3, we show the results of the reconstruction algorithm for the "house" image. The high threshold value of Canny's hysteresis threshold is chosen in an empirical way as a factor $3 / 2$ of median-absolute-deviation (MAD) at each scale. The low threshold value is then adapted such that the number of wavelet coefficients for the primal sketch at scale $i$ remains limited at $15 \%$. After 30 iterations, the reconstruction algorithm reaches good quality (PSNR 35.8dB).

\section{CONCLUSION}

The new wavelet design was based on the complex gradient-Laplace operator. First, we obtained a complexvalued basis for $L_{2}\left(\mathbb{R}^{2}\right)$ for the operator-like wavelets. Then, the Marr wavelet pyramid is derived with limited redundancy and improved steerability. We also showed how to obtain a compact multiscale primal sketch from the proposed decomposition. Finally, a reconstruction algorithm was proposed that allows to recover a good approximation of the original image from its wavelet primal sketch.

\section{APPENDIX A. PROJECTION OPERATOR $P_{\mathcal{R}}$}

The projector $P_{\mathcal{R}}$ transforms a pyramid $\left(d_{i}^{\prime}[\mathbf{k}]\right)_{i, \mathbf{k}} \in \mathcal{M}$ into the closest sequence $\left(d_{i}^{\prime \prime}[\mathbf{k}]\right)_{i, \mathbf{k}} \in \mathcal{R}$ with respect to the regularizing norm (13). We define the update term $\epsilon_{i}[\mathbf{k}]=d_{i}^{\prime}[\mathbf{k}]-d_{i}^{\prime \prime}[\mathbf{k}]$. The coefficients $\epsilon_{i}[\mathbf{k}]$ have to be 
chosen such that: (1) the norm $\left|\left(\epsilon_{i}[\mathbf{k}]\right)_{i, \mathbf{k}}\right|_{R}^{2}$ is minimum; (2) the boundary conditions imposed by the wavelet primal sketch of $f$, denoted as $\left(\left.d_{i}\right|_{\Omega_{i}}\right)$, are satisfied with $\left.\epsilon_{i}\right|_{\Omega_{i}}=\left.d_{i}\right|_{\Omega_{i}}-\left.d_{i}^{\prime}\right|_{\Omega_{i}}$, for every scale $i$.

The minimization of $\left|\left(\epsilon_{i}[\mathbf{k}]\right)_{i, \mathbf{k}}\right|_{R}^{2}$ is solved at every scale $i$ for $\mathbf{k} \in \mathbb{Z}^{2} \backslash \Omega_{i}$. We can easily derive from (13) that the constraint

$$
\epsilon_{i}[\mathbf{k}]-\left(\lambda_{i}\left[\begin{array}{ccc}
0 & 1 & 0 \\
1 & -4 & 1 \\
0 & 1 & 0
\end{array}\right] * \epsilon_{i}\right)[\mathbf{k}]=0
$$

should hold. The solution is found by a simple numerical implementation that alternates between applying the update formula

$$
\epsilon_{i}^{\text {next }}=\frac{\lambda_{i}}{1+4 \lambda_{i}}\left(\left[\begin{array}{lll}
0 & 1 & 0 \\
1 & 0 & 1 \\
0 & 1 & 0
\end{array}\right] * \epsilon_{i}^{\text {prev }}\right)
$$

and imposing the boundary conditions. The regularization can be reduced for coarser scales; e.g., $\lambda_{i}=4^{i}$.

The projector $P_{\mathcal{R}}$ is further refined to suppress spurious edges. We denote $\Omega_{i}^{\prime}$ as the domain at scale $i$ of the wavelet primal sketch of the current estimate. We extend the boundary conditions by imposing on $\Omega_{i}^{\prime} \backslash \Omega_{i}$ that the magnitude should be reduced at the low threshold value of the Canny edge detector. Note that this associated space with the refined projector changes at each iteration, meaning that convergence is no longer guaranteed.

\section{ACKNOWLEDGMENTS}

This work was supported in part by the Swiss National Science Foundation under grants PP00P2-123438 and 200020-109415, and in part by the Center for Biomedical Imaging (CIBM) of the Geneva - Lausanne Universities and the EPFL, the foundations Leenaards and Louis-Jeantet.

\section{REFERENCES}

1. S. Mallat, A Wavelet Tour of Signal Processing, Academic Press, San Diego (CA), 1998.

2. J. G. Daugman, "Complete discrete 2-D gabor transform by neural networks for image analysis and compression," IEEE Trans. on Acoustics, Speech, and Signal Processing 36, pp. 1169-1179, July 1988.

3. D. L. Donoho, "Wedgelets: Nearly minimax estimation of edges," Annals of Statistics 27, pp. 859-897, June 1999.

4. E. J. Candès and D. L. Donoho, "Ridgelets: A key to higher-dimensional intermittency?," Phil. Trans. R. Soc. Lond. A. , pp. 2495-2509, 1999.

5. M. N. Do and M. Vetterli, "The finite ridgelet transform for image representation," IEEE Transactions on Image Processing 12, pp. 16-28, Jan. 2003.

6. N. Kingsbury, "Image processing with complex wavelets," Philos. Trans. R. Soc. London A., Math. Phys. Sci. 357, pp. 2543-2560, Sept. 1999.

7. I. W. Selesnick, R. G. Baraniuk, and N. G. Kingsbury, "The dual-tree complex wavelet transform," IEEE Signal Processing Magazine 22, pp. 123-151, Nov. 2005.

8. C. Chaux, L. Duval, and J.-C. Pesquet, "Image analysis using a dual-tree $m$-band wavelet transform," IEEE Transactions on Image Processing 15, pp. 2397-2412, Aug. 2006.

9. E. Candès, "The curvelet transform for image denoising," in Proceedings of the International Conference on Image Processing, 1, p. 7, 7-10 Oct. 2001.

10. J. L. Starck, E. J. Candès, and D. L. Donoho, "The curvelet transform for image denoising," IEEE Transactions on Image Processing 11, pp. 670-684, June 2002.

11. M. N. Do and M. Vetterli, Beyond Wavelets, ch. Contourlets. Academic Press, 2003.

12. E. LePennec and S. Mallat, "Sparse geometric image representation with bandelets," IEEE Transactions on Image Processing 14, pp. 423-438, Apr. 2005.

13. G. Peyré and S. Mallat, "Surface compression with geometric bandelets," ACM Transactions on Graphics 14(3), 2005. 
14. P. Vandergheynst and J.-F. Gobbers, "Directional dyadic wavelet transforms: Design and algorithms," IEEE Transactions on Image Processing 11, pp. 363-372, Apr. 2002.

15. V. Velisavljevic, B. Beferull-Lozano, M. Vetterli, and P. Dragotti, "Directionlets: Anisotropic multidirectional representation with separable filtering," IEEE Transactions on Image Processing 15(7), pp. 19161933, 2006.

16. R. H. Bamberger and M. J. T. Smith, "A filter bank for the directional decomposition of images: Theory and design," IEEE Transactions on Signal Processing 40, pp. 882-893, Apr. 1992.

17. E. P. Simoncelli, W. T. Freeman, and E. H. Adelson, "Shiftable multi-scale transforms," IEEE Transactions on Information Theory 38, pp. 587-607, Mar. 1992.

18. E. Simoncelli and W. Freeman, "The steerable pyramid: a flexible architecture for multi-scale derivative computation," in International Conference on Image Processing, 3, pp. 444-447, 23-26 October 1995.

19. A. A. Bharath and J. Ng, "A steerable complex wavelet construction and its application to image denoising," IEEE Transactions on Image Processing 14, pp. 948-959, July 2005.

20. P. J. Burt and E. H. Adelson, "The Laplacian pyramid as a compact image code," IEEE Transactions on Communications COM-31, pp. 532-540, Apr. 1983.

21. W. T. Freeman and E. H. Adelson, "The design and use of steerable filters," IEEE Transactions on Pattern Analysis and Machine Intelligence 13, pp. 891-906, Sept. 1991.

22. G. Granlund and H. Knutsson, Signal Processing for Computer Vision, Kluwer, Norwell, MA, 1994.

23. D. Marr and E. Hildreth, "Theory of edge detection," Proceedings of the Royal Society of London. Series B, Biological Sciences 207, pp. 187-217, Feb. 1980.

24. D. Van De Ville and M. Unser, "Complex wavelet bases, steerability, and the Marr-like pyramid," IEEE Transactions on Image Processing 17, pp. 2063-2080, Nov. 2008.

25. D. Van De Ville, T. Blu, and M. Unser, "Isotropic polyharmonic B-splines: Scaling functions and wavelets," IEEE Transactions on Image Processing 14, pp. 1798-1813, Nov. 2005.

26. C. Rabut, "Elementary m-harmonic cardinal B-splines," Numerical Algorithms 2, pp. 39-62, 1992.

27. B. Forster, T. Blu, D. Van De Ville, and M. Unser, "Shift-invariant spaces from rotation-covariant functions," Applied and Computational Harmonic Analysis 25, pp. 240-265, Sept. 2008.

28. A. P. Witkin, "Scale-space filtering," in Proc. International Joint Conference on Artificial Intelligence, pp. 1019-1023, (Karlsruhe, Germany), 1983.

29. J. J. Koenderink, "The structure of images," Biol. Cybern. 50, pp. 363-370, 1984.

30. T. Lindeberg and J.-O. Eklundh, "Scale-space primal sketch: Construction and experiments," Image and Vision Computing 10(1), pp. 3-18, 1992.

31. B. Logan, "Information in the zero-crossings of band pass signals," Bell Syst. Tech. J. 56, p. 510, 1977.

32. S. Curtis, S. Shitz, and V. Oppenheim, "Reconstruction of non-periodic two-dimensional signals from zerocrossings," IEEE Trans. Acoust. Speech Signal Processing 35, pp. 890-893, 1987.

33. Y. Zeevi and D. Rotem, "Image reconstruction from zero-crossings," IEEE Acoust. Speech Signal Processing 34, pp. 1269-1277, 1986.

34. J. Sanz and T. Huang, "Theorem and experiments on image reconstruction from zero-crossings," Res. Rep. RJ5460, IBM, jan 1987.

35. R. Hummel and R. Moniot, "Reconstruction from zero-crossings in scale-space," IEEE Trans. Acoust. Speech Signal Processing 37(12), pp. 2111-2130, 1989.

36. A. Yuille and T. Poggio, "Scaling theorems for zero-crossings," IEEE Trans. Pattern Anal. Machine Intell. 8, pp. 15-25, Jan. 1986.

37. S. Mallat, "Zero-crossings of a wavelet transform," IEEE Transactions on Information Theory 37, pp. 10191033, July 1991.

38. S. Mallat and S. Zhong, "Characterization of signals from multiscale edges," IEEE Transactions on Pattern Analysis and Machine Intelligence 14, pp. 710-732, July 1992.

39. M. Unser and D. Van De Ville, "The pairing of a wavelet basis with a mildly redundant analysis via subband regression," IEEE Transactions on Image Processing 17, pp. 2040-2052, Nov. 2008.

40. D. Youla and H. Webb, "Image restoration by the method of convex projections," IEEE Trans. Med. Imaging MI-1, pp. 81-101, Oct. 1982. 